\title{
HSM Editorial Board and their Area Responsibilities (Update February 1986)
}

\author{
Name \\ Niv Ahituv \\ Peter S. Albin
}

Miriam Balaban

Tom R. Burns

Christer Carlsson

Christophe Deissenberg

Donald Gerwin

Heinz Hübner

\section{Address}

Faculty of Management

Tel-Aviv University

Tel-Aviv 69978, Israel

Center for the Study of Systems and Industrial Complexity CUNY, John Jay College 445 W. 59th Street

New York, NY 10019, U.S.A.

IFSEA, P.O.B. 2039

Rehovot 76120

Israel

Department of Sociology

Uppsala University, Box 513

S-75120 Uppsala, Sweden

FEI, Åbo Swedish University

School of Economics

Henriksgatan 7

20500 Åbo 50, Finland

Merriam Laboratory for Analytical

Political Research (MLAPR)

University of Illinois

361 Lincoln Hall, 702 S. Wright

Urbana, IL 61802, U.S.A.

School of Business Administration University of Wisconsin, P.O. Box 413 Milwaukee, WI 53201, U.S.A.

Department of Management

University of Innsbruck

Innrain 52

A-6020 Innsbruck, Austria

\section{Area Responsibility}

Distributed Information Systems; MIS; Information Processing

Human Resources and Work Organization; Automation Impacts

Information Systems; Editing and Publishing; International Cooperation between Scientific Organizations

Actor-system theory; Social Rule Systems; Sociotechnical Systems

Decision Support Systems; Fuzzy Sets

Decision Support Systems

CAD/CAM; Robotics; Impacts of Automation on the Factory

Innovation Management;

Innovation Research

North-Holland

Human Systems Management 6 (1986) 1-3 
Asterios G. Kefalas

Manfred Kochen

Donald M. Lamberton

C. Theodore Larson

Jean-Louis Le Moigne

Barry M. Levine

Robert K. Lindsay

Edward J. Lusk

Kenneth D. Mackenzie

Rashmi Mayur

Gerhard O. Mensch

Ian I. Mitroff
College of Business Administration

University of Georgia

Athens, GA 30602, U.S.A.

MHRI, 205 Washtenaw Place

University of Michigan

Ann Arbor, MI 48109-0010, U.S.A.

tel. (313) 764-2585

Economics Department Information Research Unit

University of Queensland

St. Lucia 4067, Australia

3575 E. Huron River Drive

Ann Arbor, MI 48104, U.S.A.

GRASCE-Faculté d'Economie appliquée Université de Droit, d'Economie et des Sciences d'Aix-Marseille

5, Avenue Victor-Hugo

13100 Aix-en-Provence, France

121 West Washington St.

Suite 400

Ann Arbor, MI 48104, U.S.A.

MHRI, 205 Washtenaw Place

University of Michigan

Ann Arbor, MI 48109-0010, U.S.A.

The Wharton School, Vance Hall CS

University of Pennsylvania

Philadelphia, PA 19104, U.S.A.

Mackenzie And Company, Inc.

P.O. Box 1118

Lawrence, KS 66044, U.S.A.

Urban Development Institute

38 F Maker Tower, Cuffe Parade

Bombay 400005 , India

Inst. of Applied Mathematics \& Statistics Technical University of Munich

Arcisstr. 21

D-8000 München 2, West Germany

School of Business Administration University of Southern California

Los Angeles, CA 90007, U.S.A.
Organizational Forecasting

Managing Editor

Information Economics

Built Environments

Artificial Intelligence, Information Systems and Economics

Software Program

Utilization

Artificial Intelligence

Decision Support Systems

Organizational Design; Productivity Management
Innovation Management; Sociotechnological Systems

System Complexity and Management 
Constantin V. Negoiţă

Seev Neumann

Gerald R. Salancik

August W. Smith

Arndt Sorge

James R. Taylor

Murray Turoff

Leonard Uhr

John P. van Gigch

Malcolm Warner

Fred Wenstøp

Milan Zeleny
Department of Computer Science Hunter College, 695 Park Avenue New York, NY 10021, U.S.A.

Faculty of Management

Tel-Aviv University

Tel-Aviv 69978, Israel

Department of Business Administration University of Illinois

350 Commerce Building West

Urbana, IL 61801, U.S.A.

College of Business Administration

Texas A\&M University

College Station, TX 77843, U.S.A.

International Institute of Management Platz der Luftbrücke 2

D-1000 Berlin 42, West Germany

Canadian Workplace Automation

Research Center

1575 Shomedy Blvd.

Laval (Quebec), Canada H7V 2X2

Computerized Conferencing and

Communications Center

New Jersey Institute of Technology

323 High Street, Newark

Newark, NJ 07102, U.S.A.

Computer Sciences Department

University of Wisconsin

1210 West Dayton St.

Madison, WI 53706, U.S.A.

School of Business and Public Admin. California State University, Sacramento 6000 J Street

Sacramento, CA 95819, U.S.A.

The Management College, Henley; and Brunel University

Greenlands, Henley-on-Thames

Oxon RG9 3AU, U.K.

Norwegian School of Management

Hans Burums vei 30

Postboks 69

1341 Bekkestua, Norway

Graduate School of Business, GBA 626E Policy Coordinator Fordham University at Lincoln Center New York, NY 10023, U.S.A.
Expert Systems;

Fuzzy Reasoning

Organizational Impacts of Technology

Systems Management and Systems Theory

Technological change; Work organization and skills

Office Automation Impacts

Teleconferencing; Telecommunications

Artificial Intelligence

Book Reviews Editor Epistemology of Social Sciences

Management; Factory Automation; Industrial Sociology 